\title{
A new measurement of cosmic-ray electrons and positrons with the Large Area Telescope
}

\author{
Alberto Manfreda \\ University of Pisa \\ E-mail: albertomepi.infn.it
}

\begin{abstract}
We present an updated measurement of the cosmic-ray electron and positron spectrum between $7 \mathrm{GeV}$ and $2 \mathrm{TeV}$, based on 7 years of data collected with the Fermi Large Area Telescope (LAT). The LAT is the first space-based instrument to directly explore the region above $1 \mathrm{TeV}$. At such high energies, the shape of the spectrum can provide useful information about the origin and propagation of cosmic-ray electrons in the nearby Galactic space. The spectrum that we measure suggests the presence of a break at $50 \mathrm{GeV}$. Above $50 \mathrm{GeV}$, the spectrum is well described by a single power law with a spectral index of $3.07 \pm 0.02$ (stat+syst) \pm 0.04 (energy measurement). An exponential cutoff lower than $1.8 \mathrm{TeV}$ is excluded at 95\% CL.
\end{abstract}

35th International Cosmic Ray Conference

10-20 July, 2017

Bexco, Busan, Korea 


\section{Introduction}

We present a measurement of the inclusive cosmic-ray electron and positron (CRE) spectrum from $7 \mathrm{GeV}$ to $2 \mathrm{TeV}$ based on the data collected by the Large Area Telscope (LAT) on the Fermi mission between August 4, 2008 and June 24, 2015, which was recently published by the FermiLAT collaboration [1].

Due to the relatively short diffusion distance of CREs (a few hundred parsecs at $\sim 1 \mathrm{TeV}$ [2]), measuring their spectrum above a few hundred $\mathrm{GeV}$ can provide evidence of local CRE sources from astrophysical origin (supernova remnants and pulsar wind nebulae $[3,4,5,6,7,8]$ ) or from exotic origin (dark matter $[9,10,11]$ ) and constrain theoretical models of propagation of CRs in the nearby or local galactic space.

This work updates the previous Fermi-LAT measurement of the CRE spectrum [12], based on the first year of data, extending the energy range above $1 \mathrm{TeV}$ for the first time. It also features a more detailed characterization of the systematic uncertainties of the analysis and a new, dedicated study of the effects of the geomagnetic field on the low-energy end of the spectrum. Essential for achieving these results has been the development, by the Fermi-LAT collaboration, of a radical revision of the entire event analysis, released in June 2015 under the name of Pass 8 [13].

The CRE sample selected for this analysis contains more than $10^{7}$ events, nearly $10^{4}$ of which above $1 \mathrm{TeV}$. Such an abundant data sample allows also to put stringent limits on the presence of anisotropies in their arrival directions, as reported in a follow-up search conducted by the FermiLAT collaboration [14].

\section{Event selection}

The LAT [15] is a pair conversion telescope, designed to measure $\gamma$ rays in an energy range from a few tens of $\mathrm{MeV}$ to a few hundreds of $\mathrm{GeV}$. It is composed of three main subsystems: a silicon tracker (TKR), for measuring the direction of incident particles; a $\mathrm{CsI}(\mathrm{Tl})$ calorimeter (CAL), located below the TKR, for energy measurement; a segmented anti-coincidence detector (ACD) for charged cosmic-ray background rejection. Since electromagnetic cascades are common to both electron and photon interactions in matter, the LAT is also naturally a detector for electrons and positrons.

Similarly to what we did in [12], two independent event selections have been developed for this work: High Energy (HE), above $42 \mathrm{GeV}$, which uses events from the standard on-board filter and Low Energy (LE), from $7 \mathrm{GeV}$ to $70 \mathrm{GeV}$, which uses events from an unbiased trigger sample; the latter is pre-scaled by a factor 250 , but allows the spectrum to be extended to a region where the on-board filter is not fully efficient in accepting CRE events.

We select particles with a successfully reconstructed track, a direction within $60^{\circ}$ from the instrument boresight and which cross at least 8 radiation length of material in the CAL. We also impose minimal cuts on the quality of energy and direction measurement, in order to get rid of poorly reconstructed events. We exploit the dependence of ionization on $Z^{2}$ (where $\mathrm{Z}$ is the atomic number of the bullet) to easily tag and remove particles with $Z>1$ from the dataset, cutting on the amount of ionization produced in the TKR and in the ACD. 
We use machine-learning algorithms, namely Boosted Decision Trees (BDTs), to discriminate between electrons and protons. BDTs are able to combine the information carried by several different individual observables into a single variable, $P_{\mathrm{CRE}}$, which can then be used to select signal events. BDTs are trained on simulated Monte Carlo (MC) samples of electrons and protons interacting in the LAT. We use different BDTs optimized for different energy ranges, to account for the changes in event topology in the LAT.

Comparing the distributions of data and MC of the variables used for the training of the BDTs we found, for some of them, a significant disagreement in the position of the peak. This, in turn, lead to a poor agreement between data and simulation for $P_{\mathrm{CRE}}$, which is a rather crucial point of the analysis. In order to mitigate the issue, we derived a set of corrections as functions of energy and incidence angle for the badly reproduced variables, which we applied to data. We refer to these corrections as the Individual Variables Calibration (IVC) corrections. As shown in Figure 1, above a few hundred $\mathrm{GeV}$ the agreement between data and MC improves dramatically after the IVC correction procedure.
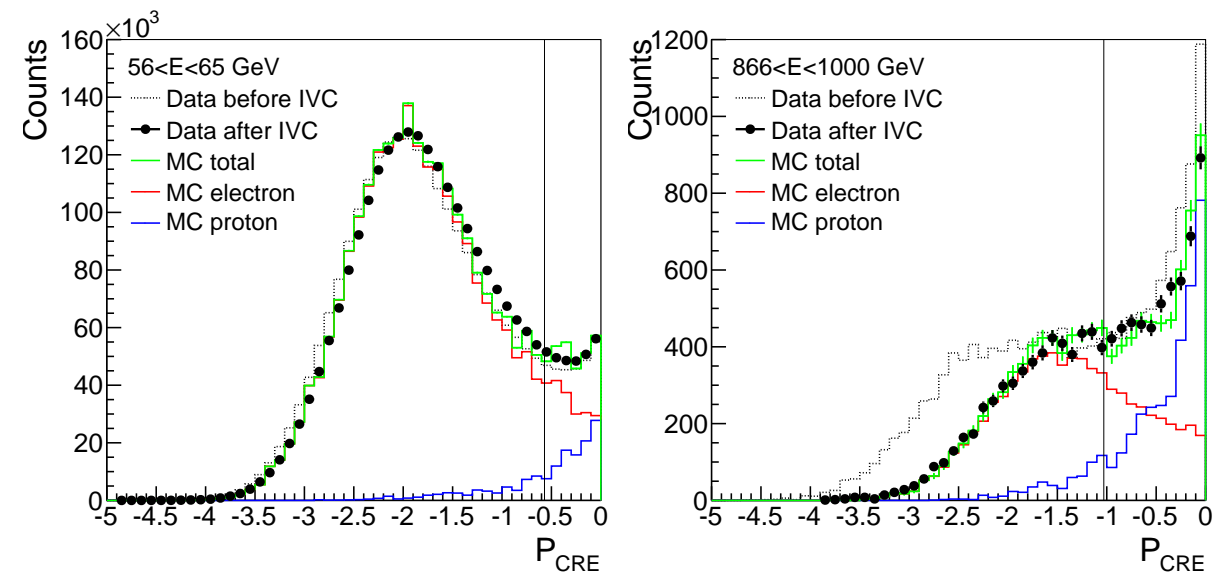

Figure 1: The result of the template fit in two energy bins [56, $65 \mathrm{GeV}]$ (left) and [866, $1000 \mathrm{GeV}]$ (right). The data $P_{\mathrm{CRE}}$ distribution is shown before (black lines) and after (black circles) IVC corrections. The green histograms correspond to the sum of the electron (red) and proton (blue) templates. The $\mathrm{x}$-axis range is chosen to focus on the electron peak region. The vertical lines show the position of the selection cut, $P_{\text {cut }}$ : events with $P_{\mathrm{CRE}}<P_{\text {cut }}$ are selected.

We estimate the residual background contamination by fitting, in each energy bin, the distribution of $P_{\mathrm{CRE}}$ in data with the sum of the MC templates for electrons and protons, letting their normalization float as free parameters for the fit. We use the renormalized MC proton template to estimate the number of background events surviving the selection, which are then subtracted from data counts to obtain the number of signal CRE events.

The acceptance and the estimated residual background contamination for the HE and LE selection are shown in Figure 2

\section{LE orbital selections}

Below $\sim 20 \mathrm{GeV}$, the spectrum of CREs observed by the LAT is strongly influenced by the 


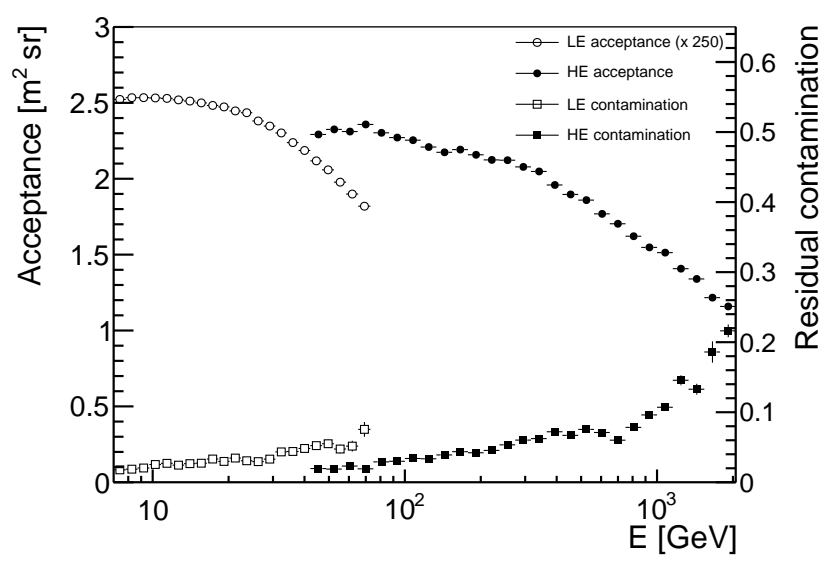

Figure 2: Acceptance and residual background contamination for the LE and HE selections. The displayed LE acceptance is multiplied by 250 (as if there were no prescale factor due to the on-board filter). For the acceptance, the energy is the true energy, while it is the measured energy for the contamination.

shielding effect of the magnetic field of the Earth. At a given geomagnetic position, only Galactic charged particles above a certain rigidity (which depends on their direction respect to the local zenith) can reach the detector. The orbit of Fermi spans an interval of vertical rigidity cut-off values from $\sim 6 \mathrm{GeV}$ to $\sim 14 \mathrm{GeV}$; in order to meaure the CRE spectrum in this energy range we selected only data collected in orbital regions in which the entire bin was above the local rigidity cutoff. For the selection, we use the McIlwain L parameter [17] to conveniently parametrize the dependence of the rigidity cut-off on geographic coordinates. The corresponding fraction of live time spent by the LAT in the selected regions is shown in Figure 3.

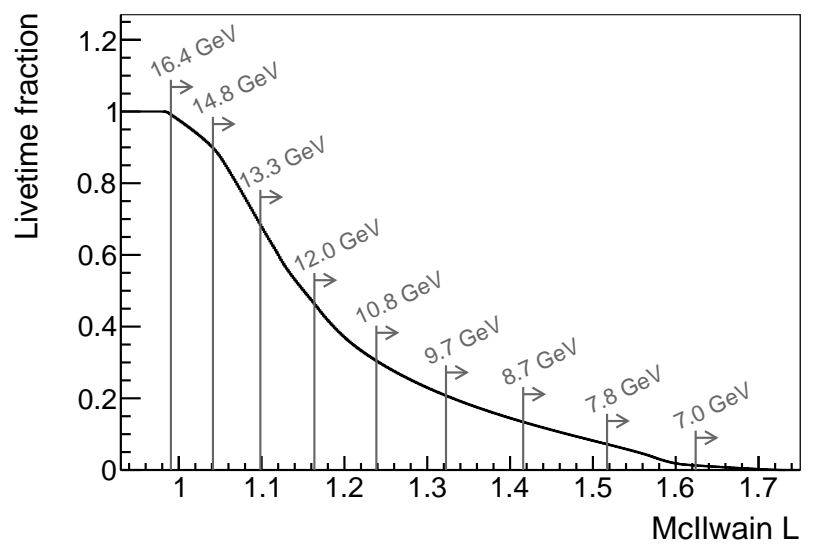

Figure 3: Normalized integral distribution of the instrument live time in different McIlwain L bins for the data sample. Grey lines show the McIlwain L cut in each energy bin (with numbers indicating the lower edge of the bin).

The cutoff is smooth and even above the nominal rigidity cutoff there is still a fraction of electrons and positrons which are prevented from reaching the detector by the magnetic shadow of the Earth. The effect is enhanced by a combination of the wide angular aperture of the LAT and its periodic rocking motion with respect to the local zenith, causing the Earth to be often very close to 
the edge of the instrument's field of view.

In order to estimate this fraction of undetected CREs and to correct the observed flux accordingly, we developed a realistic simulation of the trajectories of electrons and positrons in the orbital environment of the LAT. We use the particle trajectory tracing code developed by Smart and Shea [17] and the 2010 model of the Earth's magnetic field from the International Geomagnetic Reference Field (IGRF) [18], as we did in [19]. For a given McIlwain L selection, we use the output of the simulation to estimate the fraction of trajectories blocked by the magnetic shield of the Earth, corresponding in the real world to the fraction of unobserved CREs. The correction factors found are shown in Figure 4. We note that this effect was not explicitly accounted for in our previous measurements, though it was mitigated by the fact that the rocking angle of the instrument was smaller during the first year of the mission (after which it has been increased from $35^{\circ}$ to $50^{\circ}$ ).

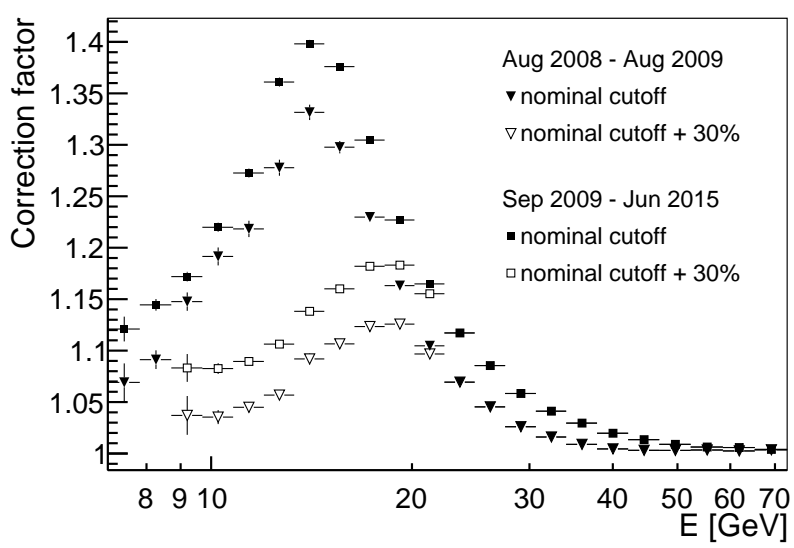

Figure 4: LE correction factors for the first year of the mission (triangles) and for the following years (squares). Full markers correspond to the nominal energy cutoff, while the empty markers correspond to a cutoff $30 \%$ higher.

\section{Systematics}

For the HE analysis we consider three sources of systematic uncertainties, related to three different parts of the event selection: the IVC corrections, the estimate of the selection acceptance and the background subtraction.

The uncertainty on the acceptance is estimated in each energy bin by measuring the variation induced in the flux by varying the cut on $P_{\mathrm{CRE}}$ around the nominal efficiency. The variation is found to be less than $2 \%$ up to $\sim 500 \mathrm{GeV}$, increasing to $6 \%$ at $2 \mathrm{TeV}$.

The uncertainty introduced by the IVC corrections procedure is estimated by deriving two alternative sets of corrections that bracket the nominal one, encompassing any residual data/MC disagreement, and recomputing the flux. It is the dominant contribute to systematics, varying from $2 \%$ at $42 \mathrm{GeV}$ to $10 \%$ at $1 \mathrm{TeV}$ and reaching $14 \%$ at $2 \mathrm{TeV}$.

As for the background subtraction, the uncertainty is driven by the imperfections of the Geant 4 simulation in predicting the fraction of protons mimicking electromagnetic showers. Based on what we found in literature $[20,21,22]$ we assume a $20 \%$ uncertainty on the estimated number of 
residual background events, leading to a change in the number of signal events of less than $2 \%$ up to $1 \mathrm{TeV}$, increasing to $7 \%$ at $2 \mathrm{TeV}$.

For the LE analysis, we consider the acceptance and contamination systematic uncertainties, as well as the changes induced in the spectrum by changing the Mcllwain L selection. The sum of these uncertainties is $\leq 4 \%$.

Regarding the systematic uncertainty on the energy measurement, we consider two independent sources of uncertainty, both of which are described in detail in [1]. In brief, the first one is the systematic uncertainty on the absolute energy scale, which does not depend on energy and is found to be $2 \%$, while the second one is the systematic uncertainty on the energy reconstruction and varies linearly with $\log _{10} E$ from $0 \%$ at $10 \mathrm{GeV}$ to $5 \%$ at $1 \mathrm{TeV}$.

As a final note, we stress the fact that the analysis is limited by systematic uncertainties across the whole energy range.

\section{Results}

Here I briefly report the most relevant results of this work. A longer discussion is given in [1], including a detailed description of how systematic uncertainties where dealt with when fitting the spectrum.

Figure 5 shows the spectra we measured with the LE and HE analysis. We note that the two spectra match very well over the overlapping range $42<E<70 \mathrm{GeV}$. Below $100 \mathrm{GeV}$, the new LAT measurement differs from the previous one by $10-30 \%$. A large part of this difference below $30 \mathrm{GeV}$ is due to the lack of correction in the previous analysis for the loss of CREs above the geomagnetic energy cutoff. After applying this correction, the remaining difference is 10-15\% and is due to imperfections in the simulation that was used in the previous analysis (remnants of electronic signals from out-of-time particles were not simulated [23]).

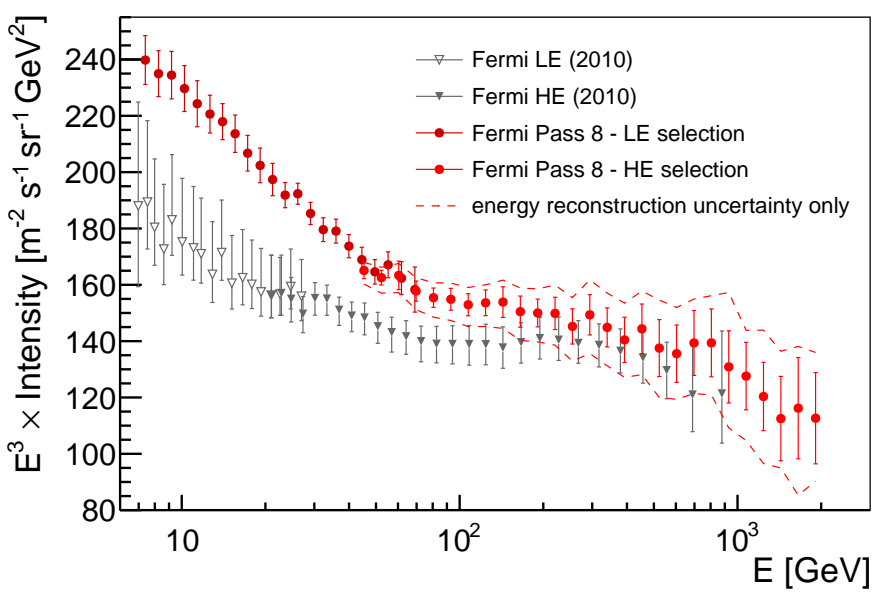

Figure 5: CRE spectrum between $7 \mathrm{GeV}$ and $2 \mathrm{TeV}$ measured by the LAT and the previous LAT measurement [12]. All error bars represent the quadratic sum of statistical and systematic uncertainties (except the one on the energy measurement). The area between the dashed lines corresponds to the uncertainty band due to the LAT energy reconstruction uncertainty only. A further $2 \%$ systematic uncertainty on the energy scale is not indicated in the plot. 
Figure 5 suggests the presence of a break in the spectrum. Indeed, a broken power-law fit to our data is preferred over a single power-law at the $4 \sigma$ level. Fitting with a broken power-law yields a break energy of $53 \pm 8 \mathrm{GeV}$ and the spectral indices below and above the break are $3.21 \pm 0.02$ and $3.07 \pm 0.02$, respectively.

Fig. 6 shows a comparison of the spectrum with other recent measurements from AMS-02 [24] and H.E.S.S. $[25,26]$. As can be seen, the LAT CRE spectrum is consistently above the AMS-02 one for energies larger than $\sim 70 \mathrm{GeV}$. The difference in spectral indices above $30.2 \mathrm{GeV}$, limit above which AMS-02 reports a single power-law spectrum, is at the level of $1.7 \sigma$. This could indicate that systematic uncertainties on the energy measurement in one or both of the results are slightly larger than estimated.

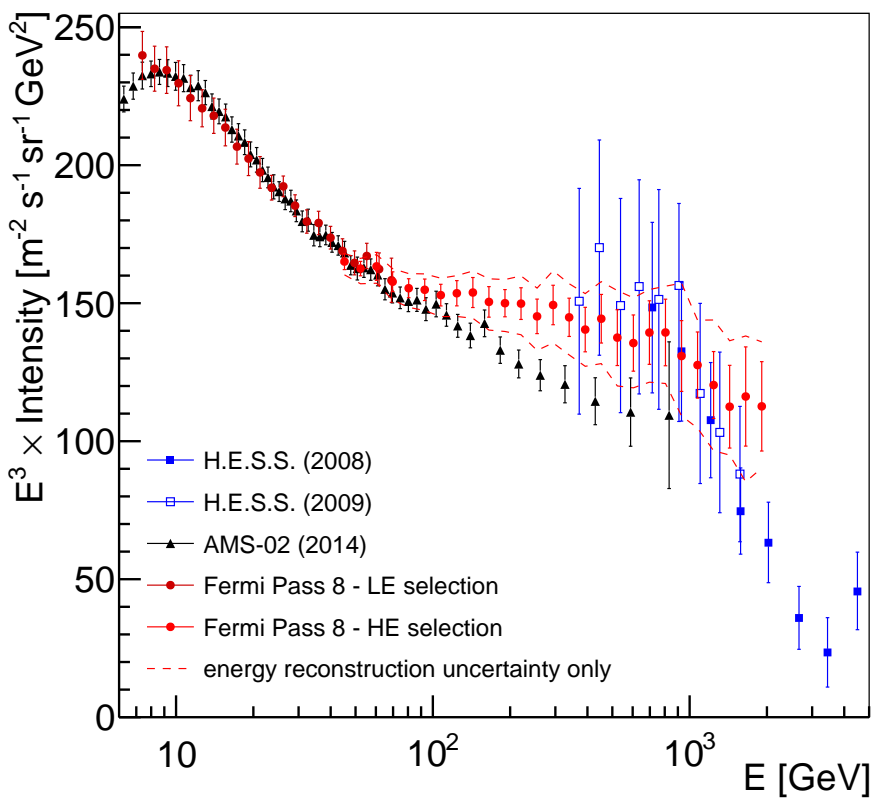

Figure 6: CRE spectrum between $7 \mathrm{GeV}$ and $2 \mathrm{TeV}$ measured by the LAT along with other recent measurements by AMS-02 [24] and H.E.S.S. [25, 26]. All error bars represent the quadratic sum of statistical and systematic uncertainties (except the one on the energy measurement). The area between the dashed lines corresponds to the uncertainty band due to the LAT energy measurement uncertainty only. A further $2 \%$ systematic uncertainty on the energy scale is not indicated in the plot.

At higher energies, H.E.S.S. reported in [25] that its data were well reproduced by an exponentially cutoff power law with a cutoff at $2.1 \pm 0.3 \mathrm{TeV}$. The LAT CRE spectrum above $50 \mathrm{GeV}$, as indicated by the previous broken power-law fits, is compatible with a single power law with a spectral index of $3.07 \pm 0.02$ (stat+syst) \pm 0.04 (energy measurement). Fitting the count spectrum above $50 \mathrm{GeV}$ with an exponentially cutoff power law $E^{-\gamma} e^{-E / E_{c}}$ does not yield statistically significant evidence for a cutoff and we exclude $E_{c}<1.8 \mathrm{TeV}$ at $95 \%$ CL.

\section{Acknowledgments}

The Fermi-LAT Collaboration acknowledges support for LAT development, operation and 
data analysis from NASA and DOE (United States), CEA/Irfu and IN2P3/CNRS (France), ASI and INFN (Italy), MEXT, KEK, and JAXA (Japan), and the K.A. Wallenberg Foundation, the Swedish Research Council and the National Space Board (Sweden). Science analysis support in the operations phase from INAF (Italy) and CNES (France) is also gratefully acknowledged.

\section{References}

[1] Abdollahi et al. (Fermi-Lat Collaboration), Phys. Rev. D, 95, (2017), 082007

[2] J. Nishimura, M. Fujii, T. Taira, International Cosmic Ray Conference 1, 488, (1979)

[3] C. S. Shen, Astrophys. J., 162, (1970), L181

[4] J. Nishimura et al., Astrophys. J., 238, (1980), 394

[5] J. Nishimura, T. Kobayashi, Y. Komori, K. Yoshida, Adv. Space Res., 19, (1997), 767

[6] F. A. Aharonian, A. M. Atoyan, H. J. Voelk, Astron. Astrophys., 294, 1995, L41

[7] T. Kobayashi, Y. Komori, K. Yoshida, J. Nishimura, Astrophys. J., 601, (2004), 340

[8] P. Blasi, Phys. Rev. Lett., 103, (2009), 051104

[9] I. Cholis, L. Goodenough, D. Hooper, M. Simet, N. Weiner, Phys. Rev. D, 80, (2009), 123511

[10] M. Cirelli, M. Kadastik, M. Raidal, A. Strumia, Nucl. Phys., B813, (2009), 1 and B873, (2013), 530

[11] L. Bergstrom, J. Edsjo, G. Zaharijas, Phys. Rev. Lett., 103, (2009), 031103

[12] M. Ackermann et al. (Fermi-Lat Collaboration), Phys. Rev. D, 82, (2010), 092003

[13] W. Atwood et al., arXiv:1303.3514

[14] S. Abdollahi et al. (Fermi-LAT Collaboration), Phys. Rev. Lett. 118, (2017), 091103

[15] W. Atwood et al. (Fermi-LAT Collaboration), Astrophys. J., 697, (2009), 1071

[16] A. Abdo et al. (Fermi-Lat Collaboration), Phys. Rev. Lett., 102, (2009), 181101

[17] D. F. Smart, M. A. Shea, Adv. Space Res., 36, (2005), 2012

[18] C. C. Finlay et al., Geophys. J. Int., 183, (2010), 1216, 1010

[19] M. Ackermann et al. (Fermi-Lat Collaboration), Astropart. Phys., 25, (2012), 346

[20] J. Yarba, J. Phys. Conf. Ser., 396 (2012), 022060

[21] A. Dotti (Geant4 Hadronic Working Group, Electromagnetic Working Group Collaboration), Proceedings of International Conference on Calorimetry for the High Energy Frontier (CHEF 2013) (2013), p. 247

[22] B. Bilki et al. (CALICE Collaboration), J. Instrum., 10, (2015) P04014.

[23] M. Ackermann et al. (Fermi-Lat Collaboration), Astrophys. J. Suppl. Ser., 203, (2012), 4

[24] M. Aguilar et al. (AMS Collaboration), Phys. Rev. Lett., 113, (2014), 221102

[25] F. Aharonian et al. (H.E.S.S. Collaboration), Phys. Rev. Lett., 101, (2008), 261104

[26] F. Aharonian et al. (H.E.S.S. Collaboration), Astron. Astrophys., 508, (2009), 561 